\title{
Long-Noncoding RNA Colorectal Neoplasia Differentially Expressed Gene as a Potential Target to Upregulate the Expression of IRX5 by miR-136-5P to Promote Oncogenic Properties in Hepatocellular Carcinoma
}

\author{
Liying Zhu a,b,c Yuyang Liu ${ }^{\mathrm{a}} \quad$ Qiuxu Chen ${ }^{\mathrm{a}}$ Gangfeng Yu $\mathrm{u}^{\mathrm{a}}$ Juan Chen ${ }^{\mathrm{a}}$ \\ Ke Chen ${ }^{\mathrm{a}}$ Nenghong Yang ${ }^{\mathrm{d}}$ Tao Zeng ${ }^{\mathrm{a}}$ Shaoying Yan ${ }^{\mathrm{a}}$ Ailong Huang ${ }^{\mathrm{a}, \mathrm{b}}$ \\ Hua Tang a
}

aKey Laboratory of Molecular Biology for Infectious Diseases (Ministry of Education), Institute for Viral Hepatitis, Department of Infectious Diseases, The Second Affiliated Hospital, Chongqing Medical University, Chongqing, ${ }^{b}$ Collaborative Innovation Center for Diagnosis and Treatment of Infectious Diseases, Zhejiang University, Hangzhou, 'Department of Medical Laboratory, Affiliated Hospital of Guizhou Medical University, Guiyang, Guizhou, 'Department of Hepatobiliary Surgery, Affiliated Hospital of Guizhou Medical University, Guiyang, Guizhou, China

\section{Key Words}

$\mathrm{HCC} \cdot$ Oncogenic property $\bullet$ CRNDE $・$ IRX5 $\bullet$ miR-136-5P

\begin{abstract}
Background/Aims: The long-noncoding RNA colorectal neoplasia differentially expressed (CRNDE) gene was first found to be activated in colorectal neoplasia. Now, it also has been found to be upregulated in many other solid tumors. Whether CRNDE affects tumorigenesis remains unknown. Methods: We conducted bioinformatics, real-time polymerase chain reaction (PCR), Western blot analysis, cell proliferation assay, colony formation assay, wound healing assay, cell migration and invasion assays, RNA immunoprecipitation, and reporter vector construction and luciferase assays. Results: CRNDE was upregulated in hepatocellular carcinoma (HCC). The overexpression of CRNDE promoted HCC cellular proliferation, migration, and invasion in intro and in vivo, and acted as an oncogene in HCC progression. Furthermore, CRNDE impaired miR-136-5P expression in a RISC manner, and a reciprocal repression feedback loop was possible between CRNDE and miR-136-5P. We found that the neighboring mRNA of CRNDE was IRX5, and IRX5 increased the tumorigenicity of HCC cells. IRX5 was a potential downstream target gene of miR-136-5P. MiR-136 regulated IRX5 by interacting with its 3 'UTR. In addition, miR-136-5P was involved in the CRNDE-regulated

L. Zhu, Y. Liu, Q. Chen and G. Yu contributed equally to this work.

\begin{tabular}{ll}
\hline Hua Tang & Department of Infectious Diseases, The Second Affiliated Hospital, Chongqing Medical University \\
and Ailong Huang & 1 Yi Xue Yuan Road, Chongqing, 400016 (China); Tel. +86 23 68486780 \\
& Fax +862368486780, E-Mail tanghua86162003@cqmu.edu.cn; ahuang1964@ cqmu.edu.cn
\end{tabular}
\end{abstract}


expression of IRX5. Conclusion: CRNDE acted as a tumor oncogene by exhibiting oncogenic properties of human HCC and revealed a novel CRNDE-miR-136-5P-IRX5 regulatory network in HCC. CRNDE may be considered to be a potential target for HCC therapies based on its ability to upregulate IRX5, and it deserves further investigation.

(C) 2018 The Author(s)

Published by S. Karger AG, Basel

\section{Introduction}

Hepatocellular carcinoma (HCC) is the most prevalent subtype of liver cancer, particularly in Asian areas. Despite the therapeutic advances in HCC in the past few decades, HCC continues to have an extremely high death rate [1].

Long-noncoding RNAs (lncRNAs) are defined as transcripts longer than 200 nucleotides that are 5'capped and 3'polyadenylated, but this class of transcripts has limited coding potential. LncRNAs play a significant role in a wide range of biological processes and can regulate gene expressions in cis or in trans by diverse mechanisms [2]. The expression of lncRNAs has been observed to vary spatially and temporally by disease, tissue, and developmental stage, indicating specific functions for lncRNAs in human development and diseases, including cancers [3-5].

The colorectal neoplasia differentially expressed (CRNDE) gene initially was identified as an lncRNA in colorectal cancer, which is significant in the proliferation, migration, and invasion of colorectal tumor cells [6]. In addition, CRNDE is overexpressed in a variety of other tumor cells, such as lung adenocarcinoma (ADC) and gastric cancer cells [7-9]. CRNDE is also the most highly expressed IncRNA in HCC, suggesting that CRNDE may be involved in the development and biological behavior of HCC $[10,11]$.

A survey within the GENCODE project found that almost 3\% of lncRNAs show a highly positive correlation with their neighboring mRNA [12]. Iroquois homeobox protein 5 (IRX5) mRNA, which is transcribed from chromosome 16 on the strand opposite to the adjacent CRNDE gene, is a member of the iroquois homeobox gene family. A previous study analyzed the correlation between IncRNA CRNDE and IRX5 mRNA expression in colorectal cancer, but little information was gained about the correlation between IncRNA CRNDE and IRX5 mRNA expression in HCC. Therefore, further investigation is needed to determine the relationship between IncRNA CRNDE and IRX5 mRNA in HCC.

LncRNA may function as a competing endogenous RNA (ceRNA) or as a molecular sponge in modulating the expression and biological functions of miRNA, such as posttranscriptional regulation, suggesting that an inverse correlation may exist between the expression of lncRNA and miRNA $[13,14]$. It remains unclear, however, whether CRNDE affects tumorigenesis by regulating miRNAs.

In this study, we investigated the expression and role of CRNDE in HCC. We demonstrated that CRNDE levels are remarkably upregulated in HCC tissues. CRNDE promoted HCC cell growth, migration, and invasion, implying a possible role of tumor oncogenic lncRNA in HCC progression. More importantly, we found that miR-136-5P negatively regulated CRNDE by directly targeting the miRNA-binding site in the body of CRNDE. Additionally, CRNDE acted as a molecular sponge for miR-136 and regulated IRX5.

\section{Materials and Methods}

\section{Cell culture and human tissue samples}

HCC cell lines SMMC7721, SK-hep1, Huh7, and HepG2; human immortalized normal human liver cell line (L02); and the embryonic kidney cell line 293T were obtained from the Chinese Academy of Sciences Cell Bank. We cultured the cell lines in Dulbecco's Modified Eagle Medium of high glucose with 10\% fetal bovine serum (FBS, BI, ISR). All of the cells were incubated at $37^{\circ} \mathrm{C}$ in a humidified incubator with $5 \%$ carbon dioxide $\left(\mathrm{CO}_{2}\right)$. We obtained 12 pairs of primary HCC and adjacent nontumor tissues from patients undergoing surgery at the Affiliated Hospital of Guizhou Medical University (Guizhou, China). We collected 
and processed the fresh tissue samples within $10 \mathrm{~min}$. Each sample was snap-frozen in liquid nitrogen and then stored at $-80^{\circ} \mathrm{C}$. The data do not contain any information that could identify the patients. All of the patients provided written informed consent, and the Committees for Ethical Review of Research involving the Affiliated Hospital of Guizhou Medical University granted ethical consent.

\section{Transient transfection}

We performed transfections using a Lipofectamine 3000 kit (Invitrogen, Carlsbad, CA, USA) according to the manufacturer's instructions. We transfected double-stranded microRNA oligonucleotides mimic or inhibitors and their respective negative control RNAs into cells at 75 pmol per well of a six-well plate according to the manufacturer's instructions, and transfected 2.5- $\mu$ g plasmids per well for DNA. The microRNA mimic and inhibitor were purchased from GenePharma (Shanghai, China). We harvested the cells $48 \mathrm{~h}$ after transfection. The sequences of the RNA used in transfections are indicated in Table 1.

\section{Reverse transcription and quantitative real-time polymerase chain reaction}

We extracted total RNA from cells using Trizol reagent (Life Technologies Corporation, Carlsbad, CA, USA) and determined RNA concentration and quality by the $260 / 280 \mathrm{~nm}$ ratio using a Nanodrop Spectrophotometer (ND-2000, Thermo Fisher Scientific, Waltham, MA, USA). FastStart essential DNA Green Master (Roche, Indianapolis, IN, USA) was used for quantitative real-time polymerase chain reaction (qRT-PCR). We used U6 and glyceraldehyde 3-phosphate dehydrogenase (GAPDH) as internal controls and expressed all of the results as the means \pm standard deviation (s.d.) of at least three independent experiments. Comparative quantification was determined using the $2^{-\Delta \Delta c t}$ method. The primers used are presented in Table 1.

\section{Cell proliferation assay}

We performed cell proliferation assays using CellTiter $96 \AA$ Aqueous One Solution Cell Proliferation Assay (MTS; Promega, Madison, WI, USA). After transfection, we seeded cells in 96-well plates at the density of $2000 /$ well. We added $20 \mu \mathrm{L}$ of MTS per well after $72 \mathrm{~h}$ and incubated the cells at $37^{\circ} \mathrm{C}$ for $2 \mathrm{~h}$. We measured the absorbance at $490 \mathrm{~nm}$.

\section{Colony-formation assay}

We seeded cells $24 \mathrm{~h}$ post-transfection into six-well plates $\left(1 \times 10^{3}\right.$ cells/well $)$ and cultured the cells for 14 days. We visualized colonies by a crystal violet cell colony staining kit and counted the number of colonies.

\section{Wound-healing assay}

We seeded cells in 6-well plates at a density of $5 \times 10^{5}$ cells. Upon reaching confluence, the cells were serum starved in FBS-free medium for $12 \mathrm{~h}$. We produced a wound by scraping across the cell monolayer using a $200-\mu \mathrm{l}$ sterile polystyrene micropipette tip. The cells were cultured and allowed to migrate into the denuded area for $48 \mathrm{~h}$. We photographed the wounds adjacent to the lines before 48 $\mathrm{h}$ after scratching under a phase contrast microscope using a $20 \times$ objective lens. We counted the cells using ImagePro Plus 6.0 software in the marked area between the labeled lines and the wound.

Table 1. Real-time PCR, construction plasmid primer, and sequences of shRNA, miRNA mimics and inhibitors

\begin{tabular}{|c|c|}
\hline Gene name & Forward primer $(\mathrm{F})$ and Reverse Primer $(\mathrm{R})$ \\
\hline \multirow{2}{*}{ CRNDE } & F: ATATTCAGCCGTTGGTCTTTGA \\
\hline & R: TCTGCGTGACAACTGAGGATTT \\
\hline \multirow{2}{*}{ GAPDH } & F: GGTCTCCTCTGACTTCAACA \\
\hline & R:GTGAGGGTCTCTCTCTTCCT \\
\hline \multirow{2}{*}{ U6 } & F:GCTTCGGCAGCACATATACTAAAAT \\
\hline & R:CGCTTCACGAATTTGCGTGTCAT \\
\hline \multirow{2}{*}{ IRX5 } & F: ACCCAGCGTACCGGAAGAA \\
\hline & R: CGGCGTCCACGTCATTTTAT \\
\hline miR-136-5P & GCCCGCCACTCCATTTGTTTTGATGATGGA \\
\hline \multicolumn{2}{|c|}{ Construction plasmid primer } \\
\hline PGL3-CRNDE & $\begin{array}{l}\text { F: GCTCTAGA CAAAGTGCTCGAGTGGTTTA } \\
\text { R: GCTCTAGA CAGGTATAAACATTCATTCAATCC }\end{array}$ \\
\hline PGL3-IRX5 3'UTR & $\begin{array}{l}\text { F: GCTCTAGATAGA TGGCCTTGGCAGTTATTTTTCC } \\
\text { R: GCTCTAGAAGA GACCAAGGCAATGGACAGTTTAT }\end{array}$ \\
\hline \multicolumn{2}{|c|}{ Sequences of shRNA clone, siRNAs, miRNA mimics and inhibitors } \\
\hline \multirow{3}{*}{ sh- CRNDE } & F: GATCCGGTGTTAAGTGTGATGCTTCCCTTCCTGTCAG \\
\hline & $\begin{array}{c}\text { AGGAAGCATCACACTTAACACCTTTTTg } \\
\text { R: AATTCAAAAAGGTGTTAAGTGTGATGCTTCCTCTGAC }\end{array}$ \\
\hline & AGGAAGGGAAGCATCACACTTAACACCg \\
\hline \multirow{3}{*}{ sh-NC } & $\begin{array}{c}\text { F:GATCCGATGAAATGGGTAAGTACATTCAAGAGATGTAC } \\
\text { TTACCCATTTCATCTTTTTG }\end{array}$ \\
\hline & R:AATTCAAAAAGATGAAATGGGTAAGTACATCTCTTGAA \\
\hline & $\begin{array}{c}\text { TGTACTTACCCATTTCATCG } \\
\text { F:GATCCGCGTACAGCACCAGCGTCATTCTTCCTGTCAGAAATGA }\end{array}$ \\
\hline sh-IRX5 & $\begin{array}{c}\text { CGCTGGTGCTGTACGCTTTTTg } \\
\text { R:AATTCAAAAAGCGTACAGCACCAGCGTCATTTCTGACAGGAAGA }\end{array}$ \\
\hline \multirow{2}{*}{ hsa-miR-136-mimic } & $\begin{array}{l}\text { ATGACGCTGGTGCTGTACGCg } \\
\text { F: ACUCCAUUUGUUUUGAUGAUGGA }\end{array}$ \\
\hline & R: UCCAUCAUCAAAACAAAUGGAGU \\
\hline \multirow{2}{*}{ Negative control(NC) } & F: GUACCUGACUAGUCGCAGATT \\
\hline & R:UCUGCGACUAGUCAGGUACTT \\
\hline \multirow{2}{*}{$\begin{array}{l}\text { has-miR-136-inhibitor } \\
\text { Negative control } \\
\text { inhibitor(NC-in) }\end{array}$} & UCCAUCAUCAAAACAAAUGGAGU \\
\hline & CAGUACUUUUGUGUAGUACAA \\
\hline
\end{tabular}




\section{Cellular Physiology Cell Physiol Biochem 2018;50:2229-2248 \begin{tabular}{l|l|l|l} 
and Biochemistry 10.1159/000495084 & $\begin{array}{l}\text { C) } 2018 \text { The Author(s). Published by S. Karger AG, Basel } \\
\text { www.karger.com/cpb }\end{array}$
\end{tabular}

\section{Cell migration and invasion assay}

We used 24-well chambers with an 8- $\mu$ m pore size (Costar 3422; Corning, NY, USA) in cell migration and invasion assays. Cells $\left(5 \times 10^{4}\right)$ in $100 \mu \mathrm{l}$ of serum-free media were seeded into the upper chamber either without or precoated with $500 \mathrm{ng} / \mathrm{ml}$ Matrigel solution (BD; Franklin Lakes, NJ, USA) in migration or invasion assay separately, and we placed $600 \mu \mathrm{L}$ of $10 \%$ FBS medium in the lower chamber. After 48 $\mathrm{h}$ of incubation, we removed the upper chambers from the plates and wiped the cells on the top side of the chamber with a cotton swab. We fixed migrating or invading cells and then used Giemsa staining. We counted five random fields under a microscope and took photos.

\section{Western blot analysis}

We extracted total proteins from the cells using radioimmunoprecipitation assay (RIPA) buffer with protease inhibitors (Beyotime, Shanghai, China) on ice, subjected to sodium dodecyl sulfate polyacrylamide gel electrophoresis and electrophoretically transferred to polyvinylidene fluoride membranes (Merck Millipore, MA, USA). Membranes were incubated in 5\% nonfat milk dissolved in tris-buffered saline (TBS) containing $0.1 \%$ Tween-20 for $2 \mathrm{~h}$ at room temperature and then incubated with primary antibodies as follows: IRX5 (1:500, Abcam, Cambridge, MA, USA), GAPDH (1:2000, Proteintech, Wuhan, China), followed by incubation with appropriate correlated horseradish peroxidase (HRP)-conjugated secondary antibody. Then, the membranes were incubated with secondary antibodies (Proteintech, Wuhan, China) at room temperature for $2 \mathrm{~h}$. Immunoblots were visualized by enhanced chemiluminescence (ECL kit; Advansta, Menlo Park, CA, USA) and scanned using ECLTM chemiluminescence detection system (Pierce Biotechnology, Waltham, MA, USA).

\section{RNA immunoprecipitation}

We lysed SMMC7721 cells by a complete RNA lysis buffer with protease inhibitor and RNase inhibitor from an EZ-Magna RIP RNA-binding protein immunoprecipitation kit (Millipore, Billerica, MA, USA) according to the manufacturer's protocol. We incubated whole cell lysate of the control groups and antimiR-136-5P groups with RNA-binding protein immunoprecipitation (RIP) buffer containing magnetic beads conjugated with human anti-Argonaute2 (Ago2) antibody (ab57113, Abcam, Cambridge, MA, USA), and negative control normal mouse immunoglobulin G (IgG; Proteintech, Wuhan, China). Samples were incubated with proteinase $\mathrm{K}$ buffer and then the immunoprecipitated RNA was isolated. We measured the RNA concentration using a NanoDrop (Thermo Fisher Scientific) and assessed the RNA quality using a bioanalyser (Agilent, Santa Clara, CA, USA). Furthermore, we obtained and analyzed the purified RNA by qRT-PCR to demonstrate the presence of the binding targets using the respective primers mentioned earlier.

\section{Construction of stable cell lines}

To obtain cell lines stably expressing CRNDE, we transfected the SMMC7721 cells with the plasmid pcDNA3.1-CRNDE a (Genscript, Nanjing, China) and plasmid pcDNA3.1, which we selected with neomycin $(1000 \mu \mathrm{g} / \mathrm{ml})$ for 4 weeks. The short-hairpin RNA targeting human CRNDE was ligated into the pGreenPuro ${ }^{\mathrm{TM}}$ shRNA vector (System Biosciences, Palo Alto, CA, USA) according to the manufacturer's protocol. We selected transfected SMMC7721 cells were selected with puromycin $(1 \mu \mathrm{g} / \mathrm{ml})$ for 4 weeks. Selected cells were further subcloned for uniform stable cell lines. We analyzed the overexpression and silence efficiency using qRT-PCR and Western blot.

\section{Reporter vectors construction and luciferase assays}

We amplified CRNDE full-length and IRX5-3'UTR sequences by PCR and cloned the sequences into a PGL3-control (Promega) to construct luciferase reporter vector (CRNDE-Wt and IRX5-Wt). We replaced the sequence of putative binding sites (miR-136-5P) as indicated by CRNDE-Mut and IRX5-Mut to mutate the putative binding site of CRNDE or IRX5. When SMMC7721 or HEK-293T cells reached 50-70\% confluence, they were seeded in 96-well plates and then were co-transfected with CRNDE-Wt (or CRNDE-Mut) or IRX5Wt (or IRX5-Mut) and miR-136-5P mimic or miR scrambled NC. We measured the luciferase activities at 48 $\mathrm{h}$ after transfection by Dual-Luciferase reporter assay kit (Promega). 


\section{Cellular Physiology Cell Physiol Biochem 2018;50:2229-2248 and Biochemistry Published online: 13 November 2018 \begin{tabular}{l|l} 
DOI: 2018 The Author(s). Published by S. Karger AG, Basel \\
www.karger.com/cpb
\end{tabular} \\ Zhu et al.: The Function of CRNDE in Hepatocellular Carcinoma}

\section{Animal studies}

The male BALB/c nude mice (4-6 weeks old) were purchased from Laboratory Animal Services Center of Chongqing Medical University. We randomly allocated 20 mice into four groups. A total of $1 \times$ $10^{7}$ SMMC7721 cells stably overexpressing CRNDE (pcDNA3.1-CRNDE) or downexpressing (sh-CRNDE) and their control cells were subcutaneously injected into the dorsal flanks of mice. We examined tumor growth at the indicated time points and measured tumor volumes by using the equation $V\left(\mathrm{~mm}^{3}\right)=\mathrm{A} \times \mathrm{B}^{2} / 2$, where $\mathrm{A}$ is the largest diameter and $\mathrm{B}$ is the perpendicular diameter. After 32 days, the mice were killed and tumors were removed and weighed. We propogatedSMMC7721 cells stably expressing CRNDE, sh-CRNDE, or negative control and injected $1 \times 10^{7}$ cells into the liver tissue of mice. After 6 weeks, the mice were killed for intrahepatic metastasis assessment.

\section{Statistical analysis}

We performed statistical analyses using SPSS 17.0 software (SPSS, Chicago, IL, USA) and assessed differences between two groups using Student's $t$ test (two-tailed). Each experiment was performed at least three times. Results of experiments are displayed as mean \pm s.d. We considered a $p$-value of $P<0.05$ to indicate statistical significance.

\section{Results}

CRNDE was upregulated in HCC and promoted HCC cell proliferation and metastasis in vitro

We first examined the expression of CRNDE in human HCC tissues and found that CRNDE was highly expressed in 12 paired HCC tissues compared with nontumor tissues (Fig. 1A). Then, we detected the expression of CRNDE in several HCC cell lines. CRNDE was also significantly upregulated in HCC cell lines (SMMC7721, Huh7, SK-Hep1, and HepG2) compared with that in immortalized, normal human liver cell line (L02) (Fig. 1B).

We examined the role of CRNDE by assessing the effect of its overexpression in HCC cells using the pcDNA3.1-CRNDE expression plasmid. CRNDE expressions in two kinds of HCC cells were drastically increased $48 \mathrm{~h}$ after transfection with pcDNA3.1-CRNDE vectors compared with cells transfected with empty pcDNA3.1 vectors (Fig. 2A). We further transfected HCC cell lines with shRNAs against CRNDE (designated as sh-CRNDE), both of which could efficiently knock down the CRNDE level (Fig. 2B). In MTS assays, overexpression of CRNDE resulted in a significant increased proliferation, whereas repressed proliferation occurred after the knockdown of CRNDE by sh-CRNDE (Fig. 1C). In colony formation assays, the ectopically CRNDE overexpressed cells displayed more colonies, yet the colony number was much lower in CRNDE-downregulated cells compared with control cells (Fig. 1D). In wound healing assays, CRNDE overexpression enhanced migratory speed; in contrast, migratory speeds were decreased after CRNDE knockdown (Fig. 1E and 1F). Transwell chamber assays confirmed the positive effect of CRNDE on HCC cell migratory capacity, and decreased cell migration after CRNDE knockdown in both SMMC7721 and HepG2 cell lines (Fig. 1G). Moreover, we investigated invasive ability alterations by using matrigelcoated transwell experiments and found more cells invading through overexpressed CRNDE, whereas knockdown of CRNDE showed the opposite effects, which was exactly in line with the expectations (Fig. 1G). Taken together, these results indicated that CRNDE increased the tumorigenicity of HCC cells in vitro.

\section{CRNDE also promoted HCC growth and metastasis in vivo}

To further probe the effect of CRNDE in vivo, we constructed four kinds of SMMC7721 cell lines: pcDNA3.1-CRNDE stably expressing CRNDE cells, pcDNA3.1 stably expressing cells, sh-CRNDE stably expressing cells, and sh-NC expressing cells. Then, we subcutaneously injected these four kinds of cells into the back of the nude mice. Compared with the pcDNA3.1 group, the pcDNA3.1-CRNDE group increased the rate of tumors formed subcutaneously (Fig. 3A). Furthermore, the final tumor weight and volume were both bigger in the CRNDE 


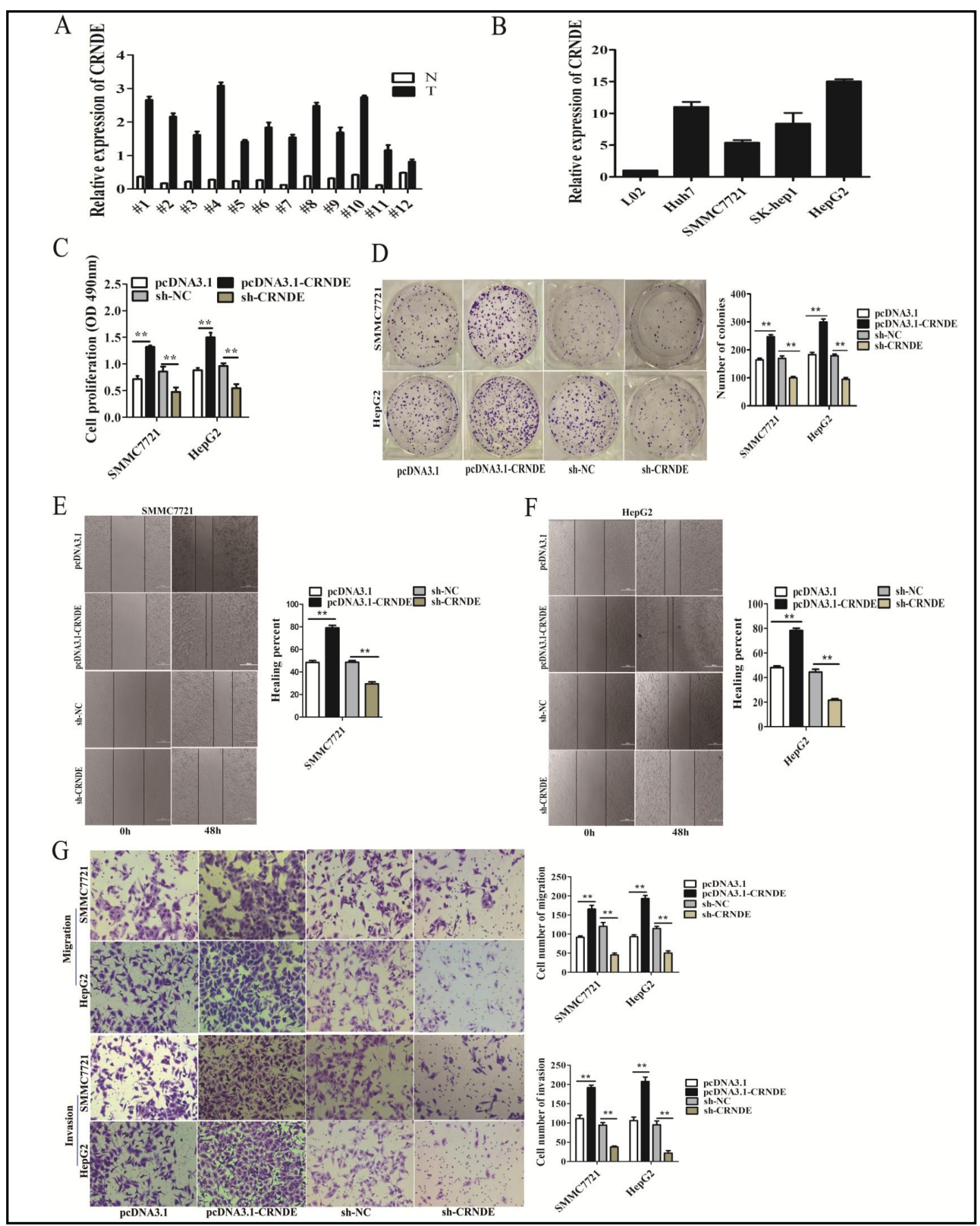

Fig. 1. CRNDE was upregulated in HCC and promoted HCC cell proliferation and metastasis in vitro. (A) CRNDE expression in 12 pairs of HCC tissues (T) and nontumor tissues (N). Transcript levels were normalized to GAPDH expression. (B) CRNDE expression in L02 cells and four HCC cell lines. Transcript levels were normalized to GAPDH expression. (C) Growth curves of SMMC7721 and HepG2 cells after transfection with pcDNA3.1-CRNDE, sh-CRNDE, or control plasmids. ${ }^{* *} \mathrm{P}<0.01$. (D) Colony formation assays determining the effect of CRNDE upregulation on the growths of SMMC7721 and HepG2 cells. Representative graphs are shown. The data graphs depict the count number of three independent experiments. ${ }^{* *} \mathrm{P}<0.01$. (E) and (F) Wound healing assay for determining the effect of CRNDE upregulation on the healing of SMMC7721 cells. ${ }^{* *} \mathrm{P}<0.01$. (G) Transwell and invasion assay of CRNDE overexpressed cells. Data are shown as mean \pm s.d. (n $=3$ ) and are representative of three independent experiments. Scale bars $=100 \mu \mathrm{m} .{ }^{* *} \mathrm{P}<0.01$ (Student's $\mathrm{t}$ test).

\section{KARGER}


Fig. 2. (A) Overexpression of CRNDE was confirmed by qRT-PCR. Transcript levels were normalized to GAPDH expression. ${ }^{* *} \mathrm{P}<0.01$. (B) Knockdown of CRNDE was confirmed with qRTPCR. Transcript levels were normalized to GAPDH expression. ${ }^{* *} \mathrm{P}<0.01$.
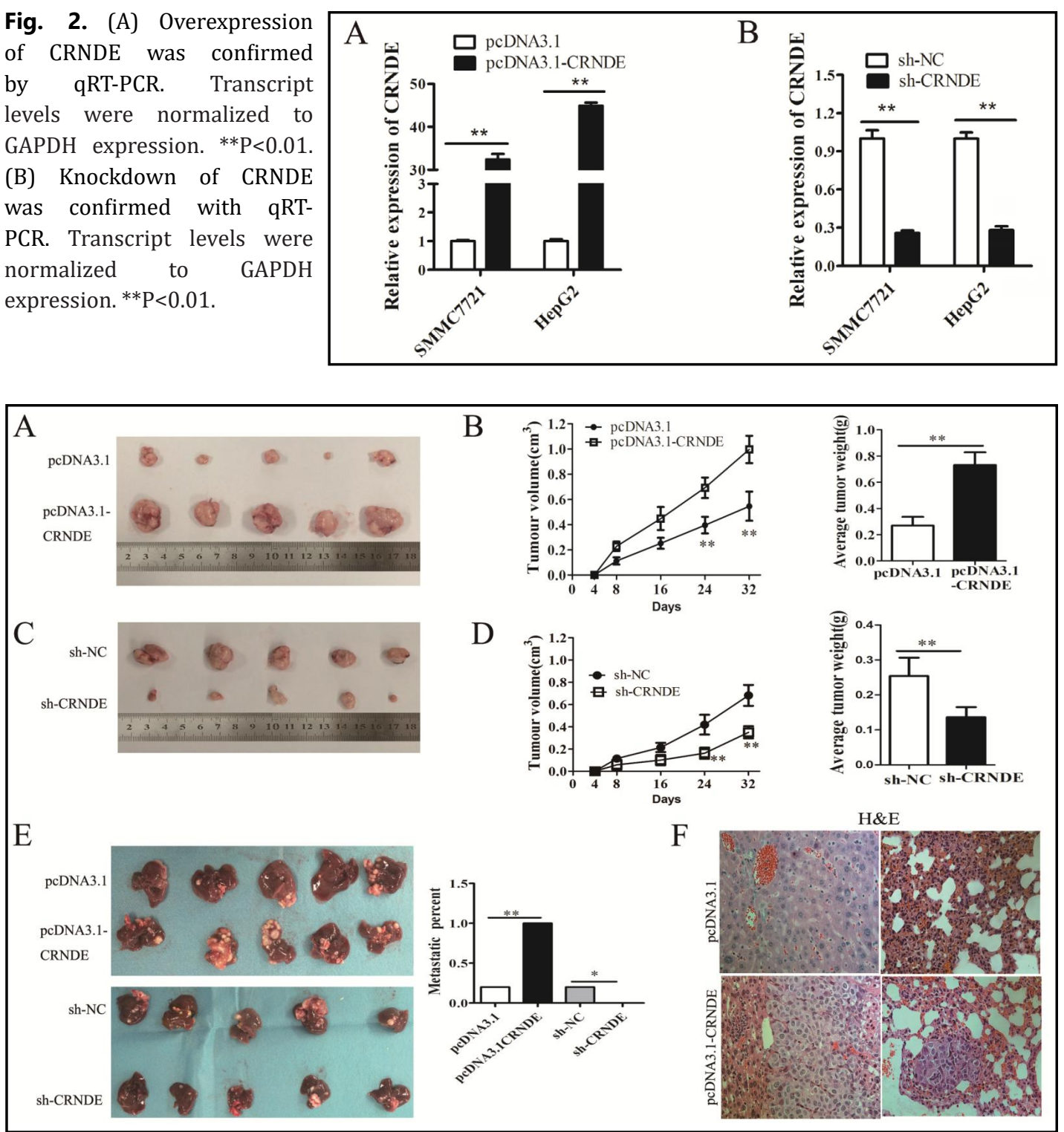

Fig. 3. CRNDE promoted HCC growth and metastasis in vivo. (A)-(D) Subcutaneous tumor model of CRNDE overexpressed SMMC7721 cells ( $\mathrm{n}=5$ for each groups). ${ }^{* *} \mathrm{P}<0.01$ (A) and (C) Representative images of tumors excised from mice 32 days after inoculation. (B) and (D) Tumor growth curves (left) and Tumor weights (right). (E) Livers of metastases 6 weeks after orthotopic implantation stably overexpressed or interference CRNDE SMMC7721 cells $(\mathrm{n}=5)$. ${ }^{*} \mathrm{P}<0.05,{ }^{* *} \mathrm{P}<0.01$. (F) H\&E staining and immunohistochemical staining of CRNDE. Representative photographs of livers and lungs.

overexpressed group than in the control group (Fig. 3B). Additionally, tumors formed by CRNDE-silencing cells grew at an obviously much slower rate than those formed by control cells (Fig. 3C), and the tumor weights and volumes from the CRNDE knockdown group were reduced significantly when compared with the control group (Fig. 3D).

Next, we tested HCC cell invasive behavior changes in orthotopic tumor models. We injected these four kinds of stable cells into the liver of nude mice and found a dramatic increase in CRNDE expression in the number of metastatic nodules in the livers of mice in the CRNDE overexpressed group ( 5 of 5 mice) compared with the vector control group ( 1 of 5 mice). Moreover, we found fewer metastatic nodules in the livers of the sh-CRNDE group ( 0 of 5 mice) compared with its control sh-NC group ( 1 of 5 mice) (Fig. 3E). Histopathological 
analysis by haemotoxylin and eosin (H\&E) staining confirmed more liver and lung metastatic foci by the overexpressed CRNDE cells (Fig. 3F). Taken together, these data demonstrated that CRNDE promoted both growth and metastasis of HCC tumors in vivo.

\section{Reciprocal repression between CRNDE and miR-136-5P}

Accumulating evidence showed that IncRNA might be a competing ceRNA or a molecular sponge in inflecting the expression and biological functions of miRNA [15]. Using a bioinformatics databases (Starbase, RNAhybrid), miR-136-5P is a putative target of CRNDE in several miRNAs (Table 2). Zheng et al. has found that CRNDE harbor miR-384 binding sites and CRNDE promotes the malignant progression of glioma by attenuating miR-384/ PIWIL4/STAT3 axis [16].

To verify our prediction that miR-136-5P could target to CRNDE, we first detected the expression of miR-136-5P in pcDNA3.1-CRNDE and sh-CRNDE cells by qRT-PCR. The expression of miR-136$5 \mathrm{P}$ decreased in the pcDNA3.1-CRNDE group compared with the pcDNA3.1 group, whereas

Table 2. StarBase(v2.0)predicted the miRNAs that target CRNDE

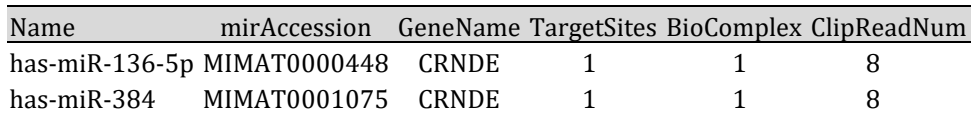

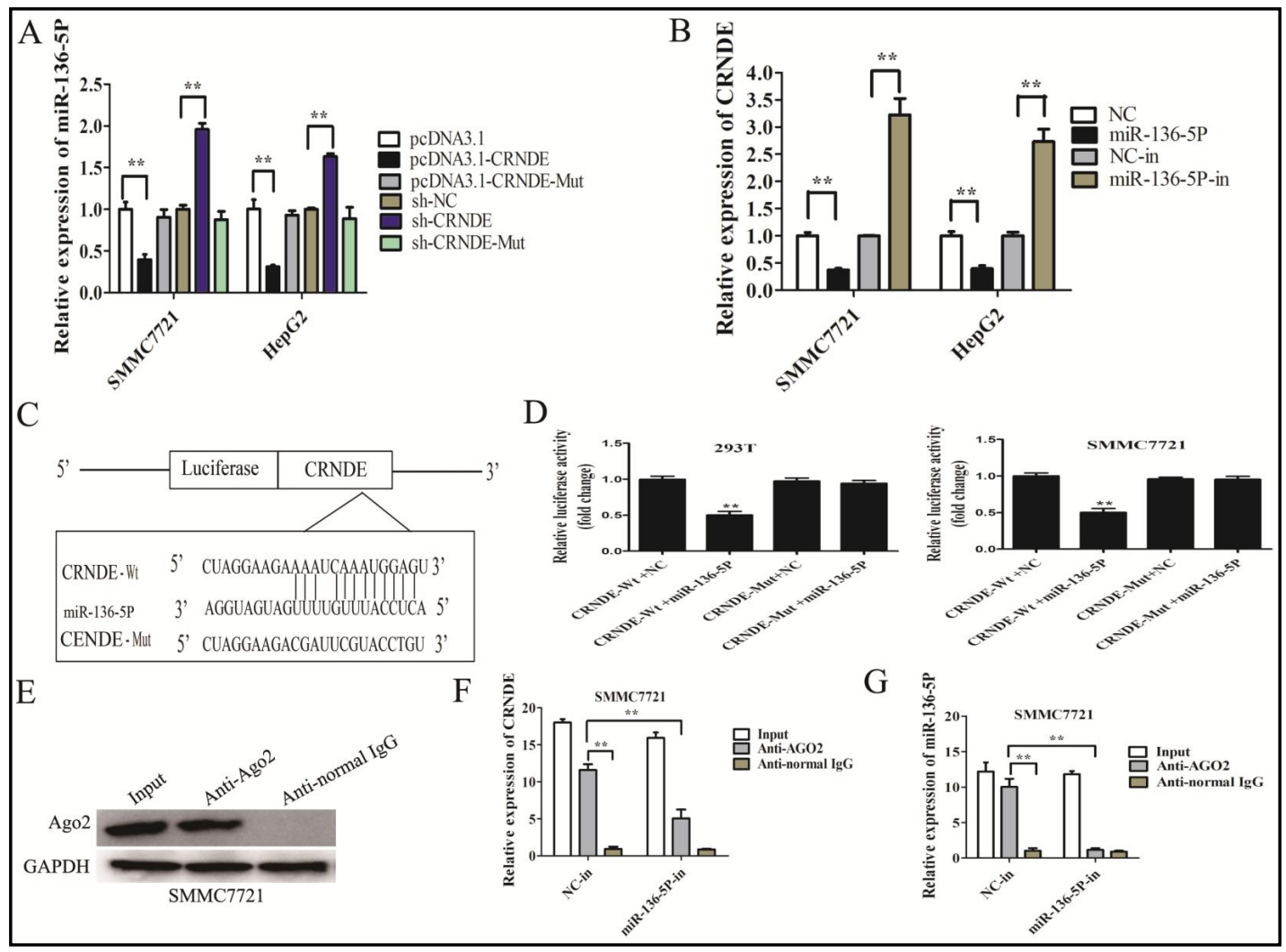

Fig. 4. Reciprocal repression between CRNDE and miR-136-5P. (A) miR-136-5P in HCC cells that stably overexpressed and knocked down CRNDE, using U6 as an internal control. ${ }^{* *} \mathrm{P}<0.01$. (B) qRT-PCR analysis of CRNDE expressions in SMMC7721 and HepG2 cells transfected with miR-136-5P mimic (miR-136-5P) or NC, using GAPDH as an internal control. ${ }^{* *} \mathrm{P}<0.01$. (C) Predicted binding sites for miR-136-5P in CRNDE sequences. Numbers show the nucleotides relative to the transcriptional start site of CRNDE. (D) Luciferase assays of 293T and SMMC7721 cells transfected with PGL3-CRNDE-Wt or PGL3-CRNDE-Mut reporter and NC or miR-136-5P mimic. ${ }^{* *} \mathrm{P}<0.01$. (E) Ago2 protein immunoprecipitated from cell extracts by Ago 2 antibody or IgG detected by Western blot analysis. (F) and (G) The amount of CRNDE and miR-136-5P bound to Ago 2 or IgG was measured by qRT-PCR in the presence of miR-136-5P inhibitor or negative control. ${ }^{* *} \mathrm{P}<0.01$. 
Fig. 5. (A) The effect of MiR-136-5P mimic was confirmed with qRTPCR. Transcript levels were normalized to U6 expression. ${ }^{*} * \mathrm{P}<0.01$. (B) The effect of miR-136-5P inhibitor was confirmed with qRT-PCR. Transcript levels were normalized to U6 expression. ${ }^{* *} \mathrm{P}<0.01$.

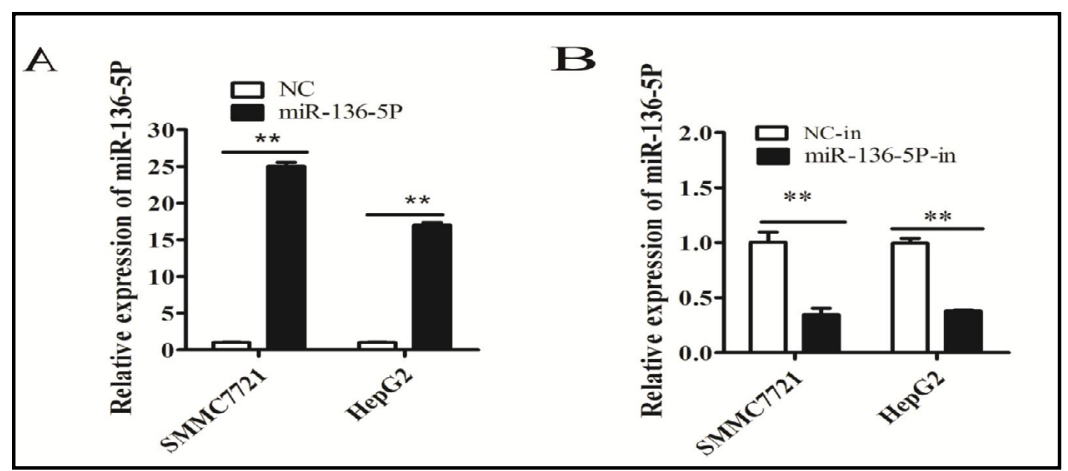

the expression of miR-136-5P increased in the sh-CRNDE group compared with the sh-NC group. We did not, however, find any significant difference in the CRNDE-Mut groups (Fig. 4A). These data showed that CRNDE could regulate miR-136-5P expression. To further analyze the relationship between miR-136-5P and CRNDE, we used miR-136-5P mimics (miR136-5P) and miR-136-5P inhibitors (miR-136-5P-in) and confirmed the overexpression or inhibition of miR-136-5P by qRT-PCR (Fig. 5A and 5B). Then, we transfected HCC cells with miR-136-5P mimics or miR-136-5P inhibitors and detected the expression of CRNDE (Fig. 4B). Bioinformatics analysis of miRNA recognition sequences revealed that miR-136$5 \mathrm{P}$ binding sites were presented in CRNDE (Fig. 4C). To explore the underlying mechanism of the lncRNA/miRNA regulatory function, we conducted dual-luciferase reporter assays to determine the binding sites of CRNDE and miR-136-5p. The luciferase activity in the CRNDE$\mathrm{Wt}+$ miR-136-5P group was significantly lower than that in the control group, whereas the luciferase activity in the CRNDE-Mut group was not affected (Fig. 4D).

Previous studies have demonstrated thatmiRNAsarepresentin miRNA ribonucleoprotein complexes (miRNPs) that contain Ago2, the key component of the RNA-induced silencing complex (RISC) $[17,18]$. To test whether CRNDE associated with miR-136-5P, RIP assay was carried out. Ago2 protein was efficiently immunoprecipitated from cell extracts by Ago2 antibody (Fig. 4E). The expressions of CRNDE and miR-136-5P both increased in the antiAgo2 group compared with the abnormal IgG group. In the miR-136-5P inhibitor group, the expressions of CRNDE and miR-136-5P immunoprecipitated with Ago2 were lower than those in the control group ( $P<0.01$; Fig. $4 \mathrm{~F}$ and $4 \mathrm{G})$. Taken together, these results suggested that CRNDE could impair miR-136-5P expression in an RNA-induced silencing complex (RISC) manner, and a reciprocal repression feedback loop might exist between CRNDE and miR-136-5P.

miR-136-5P was downregulated in HCC and functioned as tumor suppressor

qRT-PCR showed that miR-136-5P was downregulated in the 12 paired HCC tissues but not in the nontumor tissues (Fig. 6A). Compared with L02 cells, HCC cell lines displayed lower expressions of miR-136-5P (Fig. 6B). The overexpression of miR-136-5P inhibited cell growth in MTS assays, whereas promoted proliferation occurred after knockdown of miR-136-5P (miR-136-5P-in) (Fig. 6C). Transwell assays confirmed the repression of miR136-5P on HCC cell migratory capacities in both SMMC7721 and HepG2 cell lines (Fig. 6D). However, the knockdown of miR-136-5P significantly reduced the number of cells crossing the membrane (Fig. 6D). In matrigel-coated transwell assays, more cells invaded when miR136-5P was overexpressed (Fig. 6D). The knockdown of miR-136-5P showed the opposite effect (Fig. 6D). 


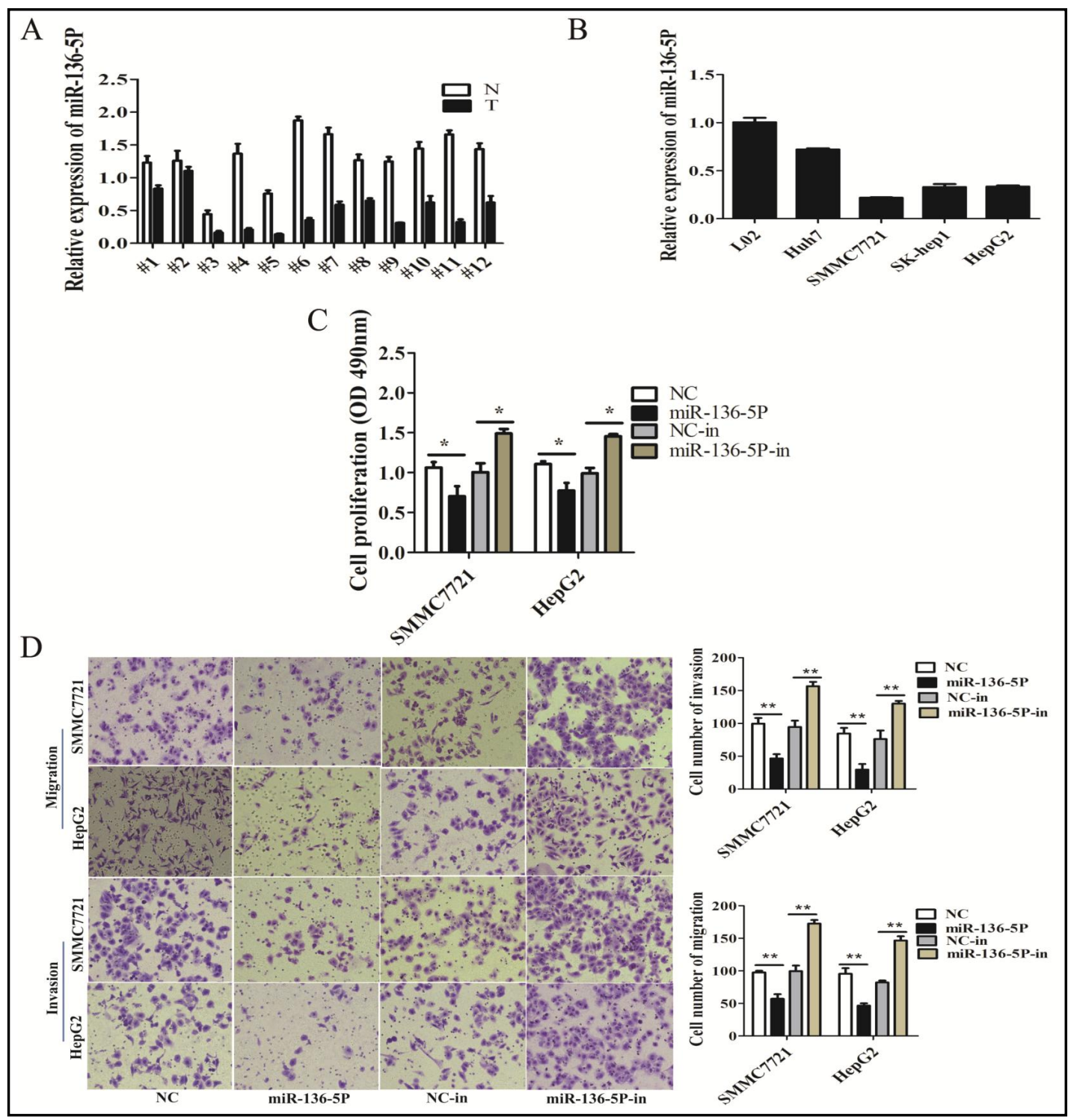

Fig. 6. MiR-136-5P was downregulated in HCC and functioned as tumor suppressor. (A) qRT-PCR analysis of miR-136-5P expressions in 12 pairs of HCC tissues (T) and nontumor tissues (N), using U6 as an internal control. (B) qRT-PCR analysis of miR-136-5P expressions in L02 cells and four cell lines, using U6 as an internal control. (C) Growth curves of SMMC7721 and HepG2 cells after transfection with miR-136-5P or miR-136-5P inhibitor (miR-136-5P-in) were determined by MTS assays. ${ }^{* *} \mathrm{P}<0.01$. (D) The effects of miR136-5P on the migration and invasion of SMMC7721 and HepG2 cells were performed using transwell assays. ${ }^{* *} \mathrm{P}<0.01$.

Overexpression of miR-136-5P largely reversed CRNDE-induced oncogenetic effects on HCC cells

To study the effects of miR-136-5P on cell proliferation, migration, and invasion mediated by CRNDE, SMMC7721 and HepG2 cells were transfected with miR-136-5P mimics and the plasmids expressing CRNDE. As shown in Fig. 7A, miR-136-5P significantly decreased the CRNDE expressions in two cell lines, but had nearly no effect on the expression of mutant miR-136-5P (Fig. 7B). MTS proliferation assays revealed that miR-136-5P suppressed cell proliferations and CRNDE promoted cell proliferations. miR-136-5P reversed the effect of CRNDE in promoting cell proliferations, when co-transfected with miR-136-5P mimics and plasmids expressing CRNDE (Fig. 7C). Moreover, transwell migration and invasion assays 


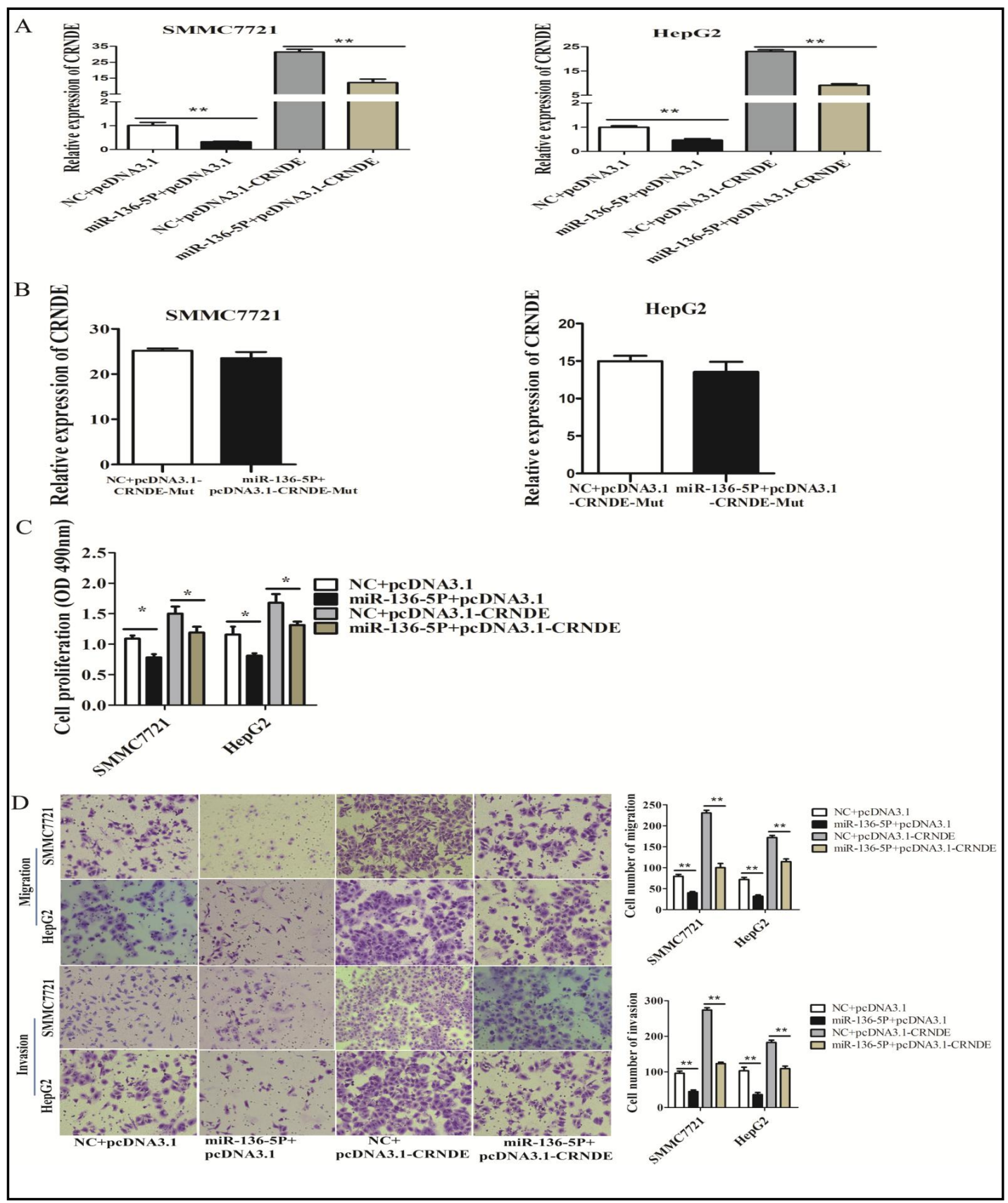

Fig. 7. Overexpression of miR-136-5P largely reversed CRNDE-induced oncogenetic effects on HCC cells. (A) SMMC77221 and HepG2 cells were co-transfected with miR-136-5P mimic and CRNDE expression plasmid, and the effect of miR-136-5P on ectopically expressed CRNDE was analyzed by qRT-PCR. ${ }^{* * P}<0.01$. (B) SMMC77221 and HepG2 cells were co-transfected with miR-136-5P mimic and pcDNA3.1-CRNDE-Mut plasmid (with miR-136-5P mutant binding site), and the effect of CRNDE on ectopically mutant CRNDE expression was analyzed by qRT-PCR. ${ }^{* *} \mathrm{P}<0.01$. (C) SMMC7721 and HepG2 cells were co-transfected with a negative control or miR-136-5P mimic and control plasmid (pcDNA3.1) or CRNDE expression plasmid (pcDNA3.1-CRNDE) and cell proliferations were determined using MTS assays. ${ }^{*} \mathrm{P}<0.05$. (D) Transwell migration and invasion assays of HCC cells after co-transfection with negative control or miR-136-5P mimic and control plasmid (pcDNA3.1) or CRNDE expression plasmid (pcDNA3.1-CRNDE). ${ }^{* *} \mathrm{P}<0.01$. 
Fig. 8. CRNDE increased the expression of IRX5. (A) CRNDE was located near IRX5 on chromosome 16. (B) qRT-PCR analysis of IRX5 mRNA expressions when SMMC7721 and HepG2 cells were transfected with pcDNA3.1CRNDE or pcDNA3.1 vectors. Transcript levels were normalized to GAPDH expression. ${ }^{* *} \mathrm{P}<0.01 . \quad$ (C) Western blot analysis of IRX5 protein expressions after SMMC7721 and HepG2 cells were transfected with pcDNA3.1-CRNDE or pcDNA3.1 vectors, using

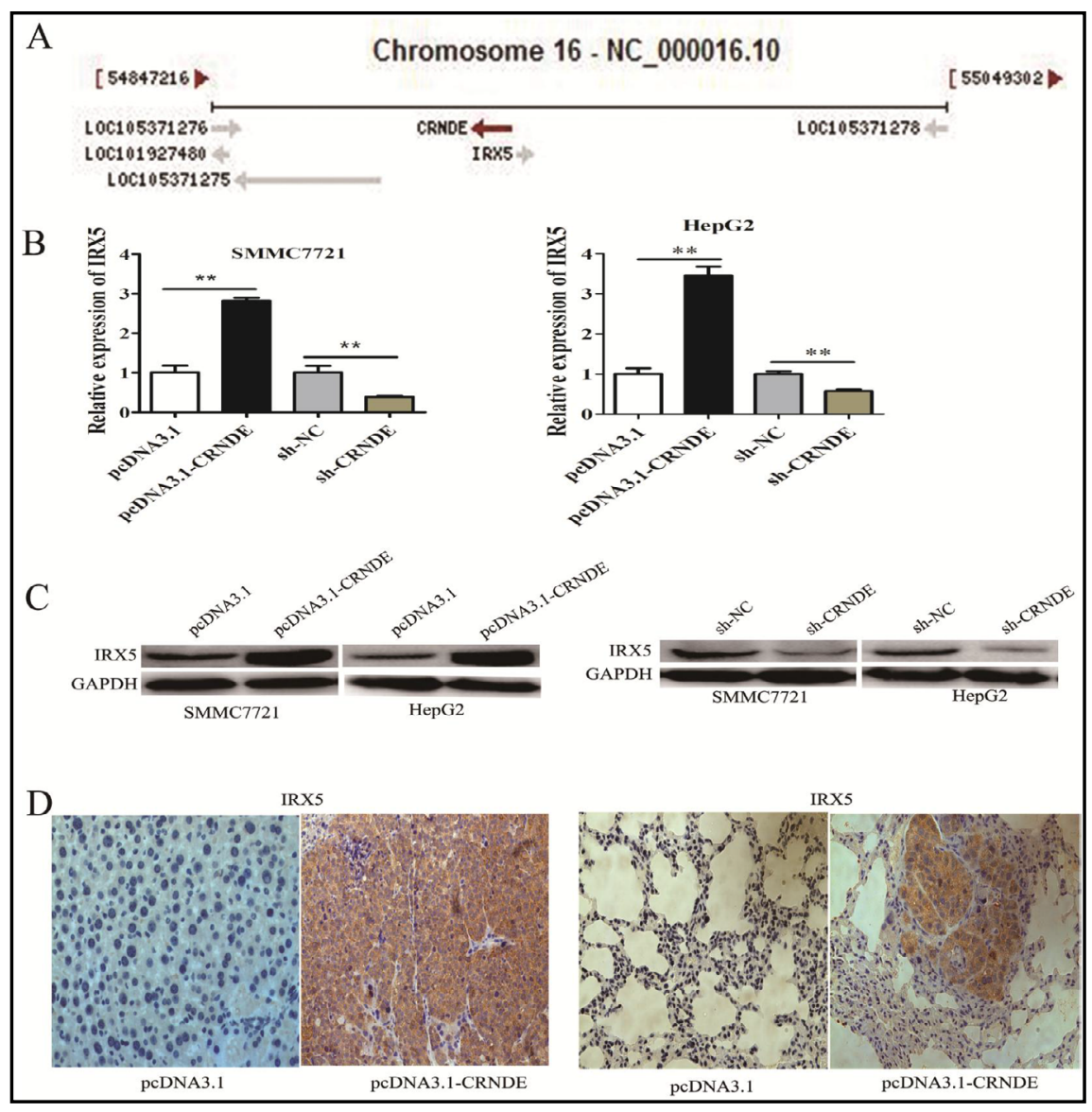
GAPDH as an endogenous control. (D) IRX5 immunohistochemical staining of mouse liver (left) and lung (right). Representative micrographs were shown, original magnification $(\times 400)$.

demonstrated that miR-136-5P suppressed and CRNDE promoted cell migration and invasion. Conversely, when co-transfected with miR-136-5P mimics and CRNDE-expressing vectors, miR-136-5P reversed cell migration and invasion promoted by CRNDE (Fig. 7D). These observations suggested that the effects of CRNDE overexpression on the promotion of SMMC7721 and HepG2 cell proliferation, migration, and invasion could be reversed by miR-136-5P.

\section{CRNDE increased the expression of IRX5}

These results illustrated that CRNDE and miR-136-5P could modulate the biological behaviors of HCC cells, but the underlying molecular mechanisms remain unclear. Our previous studies found that IncRNAs could modulate the biological behaviors of HCC cells through their neighboring mRNAs $[15,19]$. We found that the neighboring mRNA of CRNDE was IRX5 (Fig. 8A). We performed qRT-PCR and Western blot assays to confirm the effects of CRNDE on the expressions of mRNA and protein levels of IRX5. The mRNA expression levels of IRX5 in the cells transfected with pcDNA3.1-CRNDE were higher than those cells transfected with pcDNA3.1 (Fig. 8B). In contrast, the mRNA level of IRX5 in the shCRNDE group was downregulated compared with the sh-NC group (Fig. 8B). Similarly, IRX5 protein was markedly decreased in pcDNA3.1-CRNDE transfected cells than in the control cells (Fig. 8C), whereas IRX5 protein increased in sh-CRNDE transfected cells (Fig. 8C). Immunohistochemistry assay showed a strong expression of IRX5 in the liver and lung of mice in the CRNDE overexpression group compared with the control group in vivo (Fig. 8D). These results revealed that CRNDE upregulated the expression of IRX5 in vitro and in vivo. 
Fig. 9. (A) qRT-PCR analysis of IRX5 expressions in 12 pairs of HCC tissues (T) and nontumor tissues Transcript levels were normalized to GAPDH expression. (B) qRT-PCR analysis of IRX5 expressions in L02 cells and four HCC cell lines. Transcript levels were normalized to GAPDH expression. (C) Overexpression effect of IRX5 was confirmed with qRT-PCR.

Transcript levels were normalized to GAPDH expression.

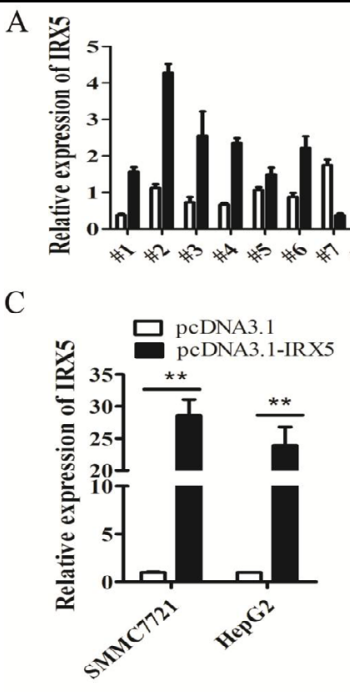

E

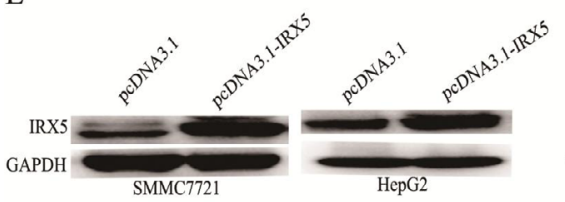

$\mathrm{B}$

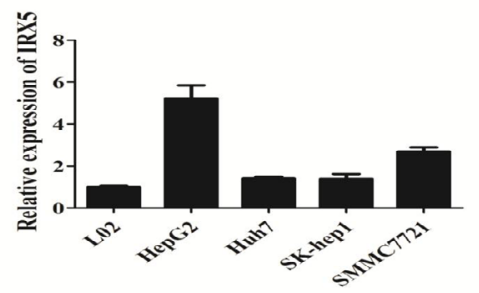

D

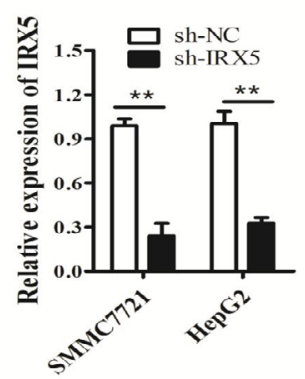

F

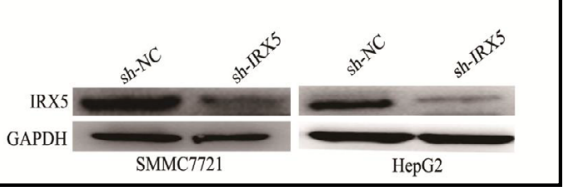

** $\mathrm{P}<0.01$. (D) Knockdown effect of IRX5 was confirmed with qRT-PCR. Transcript levels were normalized to GAPDH expression. ${ }^{* *} \mathrm{P}<0.01$. (E) Overexpression effect of IRX5 was confirmed with Western blot analysis. Transcript levels were normalized to GAPDH expression. ${ }^{* *} \mathrm{P}<0.01$. (F) Knockdown effect of IRX5 was confirmed with Western blot analysis. Transcript levels were normalized to GAPDH expression. ${ }^{* * P}<0.01$.

IRX5 was significantly upregulated in HCC and promoted HCC cell proliferation and metastasis

First, we measured the expression of IRX5 in HCC tissues and HCC cell lines by qRT-PCR and Western blot. The results showed that IRX5 were significantly higher in tumor tissues and HCC cell lines (Figs. 9A and 9B; Fig. 10A and 10B). Furthermore, the immunohistochemistry assay revealed that the IRX5 proteins were differentially expressed between human tumor tissues and their matched nontumor tissues. We detected strong immunoreactivities for IRX5 in the cell nucleus and cytoplasm of the tumor tissues. The nontumor tissues, however, showed weak or no expression of IRX5 (Fig. 10C).

Second, we examined the role of IRX5 by assessing the effects of its overexpression in HCC cells using the pcDNA3.1-IRX5 expression plasmid by qRT-PCR and Western blot. IRX5 expressions in both cells increased drastically $48 \mathrm{~h}$ after transfection with pcDNA3.1IRX5 vectors compared with cells transfected with empty pcDNA3.1 vectors. We further transfected HCC cell lines with shRNAs against IRX5 (designated sh-IRX5), both of which could efficiently knock down IRX5 expressions (Fig. 9C-9F). In MTS assays, the overexpression of IRX5 resulted in significantly increased proliferation, whereas repressed proliferation was observed after knockdown of IRX5 (Fig. 10D). Transwell chamber assays confirmed the positive effect of IRX5 on HCC cell migratory capacity, with decreased cell migration after IRX5 knockdown (Fig. 10E). In matrigel-coated transwell assays, more cells invaded when overexpressed IRX5 and knockdown of IRX5 showed the opposite effects (Fig. 10E). Taken together, these results indicated that IRX 5 increased the tumorigenicities of HCC cells. 


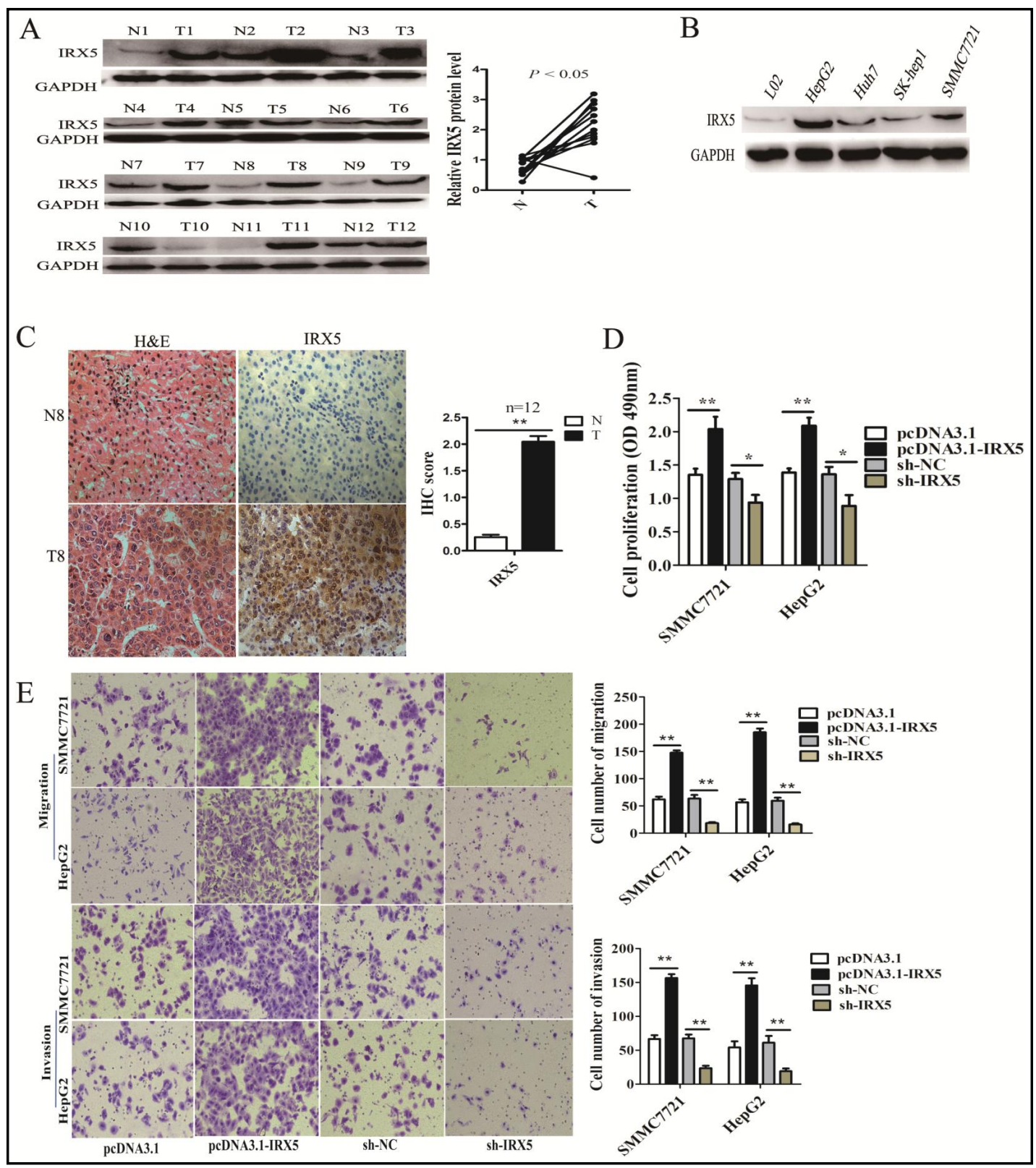

Fig. 10. IRX5 was significantly upregulated in HCC and promoted HCC cell proliferation and metastasis. (A) Western blot analysis of IRX5 protein expressions in 12 pairs of HCC tissues (T) and nontumor tissues $(\mathrm{N})$, using GAPDH as an endogenous control. Quantitation of IRX5 protein expression levels by their integrated light density values. $n=12,{ }^{*} \mathrm{P}<0.05$. (B) Western blot analysis of IRX5 expressions in L02 cells and four cell lines, using GAPDH as an endogenous control. (C) H\&E staining and immunohistochemical staining of IRX5 in HCC tissues (T) and nontumor tissues (N). No. 8 was the representative micrograph. Total immunohistochemical (IHC) score of IRX5 in HCC tissues and nontumor tissues ( $\mathrm{n}=12$ ). ${ }^{* *} \mathrm{P}<0.01$. (D) Growth curves of SMMC7721 and HepG2 cells after transfection with pcDNA3.1- IRX5, sh-IRX5 or control plasmids. ${ }^{* *} \mathrm{P}<0.01$. (E) Transwell and invasion assay of IRX5 overexpressed cells. Data are shown as mean \pm s.d. $(\mathrm{n}=3)$ and are representative of three independent experiments. Scale bars $=100 \mu \mathrm{m} .{ }^{* * P}<0.01$ (Student's t test). 
MiR-136-5P inhibited the expression of IRX5 by targeting its 3'UTR and miR-136-5P was involved in the CRNDE-regulated expression of IRX5

Interestingly, IRX5 was a potential downstream target gene of miR-136-5P predicted by a bioinformatics database (Targetscan, miRanda) (Tables 3 and 4). To experimentally verify the prediction, we transfected cells with miR-136-5P mimics or miR-136-5P inhibitors, and assessed the mRNA and protein expression levels of IRX5 by qRT-PCR and Western blot assay. As a result, miR-136-5P inhibited the expression of IRX5 in both mRNA and protein levels (Fig. 11A and B).

To further elucidate whether IRX5 was a functional target of miR-136-5P, we conducted dual-luciferase reporter assays in 293T and SMMC7721 cells. The binding sites of miR-136-5P in IRX5-3'UTR are illustrated in Fig. 11C. As shown in Fig. 11D, no significant difference occurred in the relative luciferase activity between the IRX5-3'UTR-Mut+miR-136-5P and IRX5-3'UTRMut+NC groups $(P>0.05)$, but the relative luciferase activity of the IRX5-3'UTR-Wt+miR-136$5 P$ group decreased significantly compared with the IRX5-3'UTR $-\mathrm{Wt}+\mathrm{NC}$ group $(P<0.01)$.

To clarify whether miR-136-5P was involved in the CRNDE-mediated expression of IRX5, we measured the IRX5 mRNA and protein expression levels by qRT-PCR and Western blot assay. After several complex transfections, the pcDNA3.1-CRNDE+miR-136-5P inhibitor group had a higher expression of IRX5 mRNA than the pcDNA3.1-CRNDE+miR-136-5P group $(P<0.01)$, whereas the sh-CRNDE+miR-136-5P group had a lower expression of IRX5 mRNA than the sh-CRNDE+miR-136-5P-inhibitor group $(P<0.01)$ (Fig. 11E). Similar results of IRX5 protein expressions were found in Western blot analysis (Fig. 11F). All of these results indicated that CRNDE might upregulate the expression of IRX5 by binding miR-136-5P.

\section{Discussion}

Over the past few years, the importance of IncRNA in HCC tumorigenesis and progression has been gradually recognized. Some IncRNAs, such as HULC, MALAT1, MEG3, and UCA1, have been found to be significantly elevated in HCC and could promote the proliferation and metastasis of HCC [20-22].

Microarray analysis of 454 tissue specimens (discovery) and 68 previously untested specimens (validation) showed an elevated expression of CRNDE in $>90 \%$ of colorectal adenomas and ADCs. These findings were confirmed and extended by exon microarray studies and qRT-PCR assays. CRNDE was upregulated in glioma stem cells (GSCs) and in human glioma tissues, and the overexpression of CRNDE was found to promote cellular proliferation, migration, and invasion and to inhibit apoptosis in GSCs [23]. CRNDE promoted gall bladder carcinoma carcinogenesis and served as a scaffold of DMBT1 and C-IAP1 complexes to activate the PI3K-AKT pathway [24]. Esposti found that CRNDE was upregulated in hepatocellular carcinoma using RNA sequencing [25]. LncRNA CRNDE was found to promote hepatic carcinoma cell proliferation, migration, and invasion by suppressing miR-384 10. In this study, CRNDE increased in human HCC tissues and cell lines, and CRNDE promoted HCC cell proliferation, migration, and invasion in vitro and in vivo, which is consistent with other studies. 


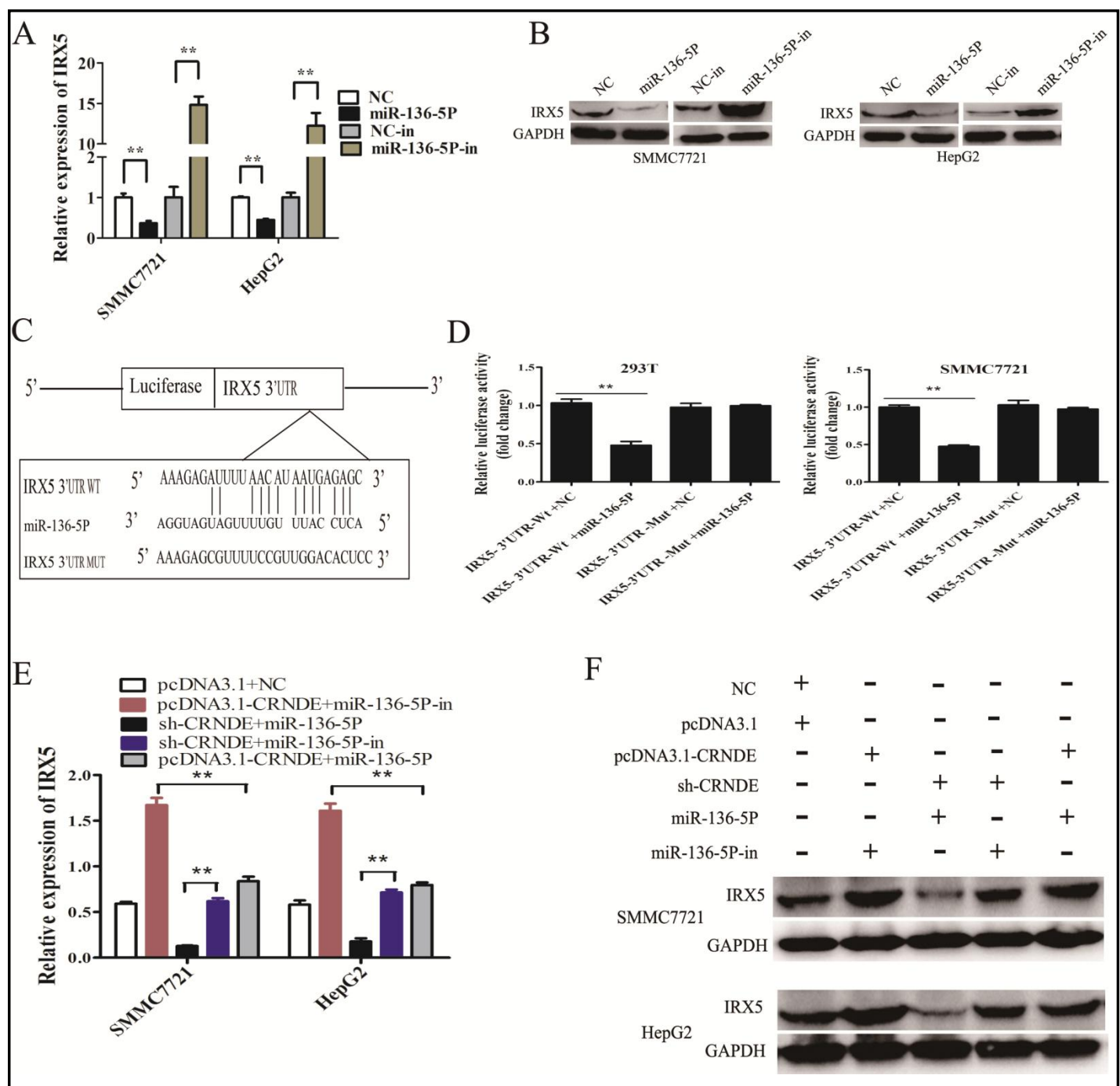

Fig. 11. MiR-136-5P inhibited the expression of IRX5 by targeting its 3'UTR and miR-136-5P was involved in the CRNDE-regulated expression of IRX5. (A) qRT-PCR analysis of IRX5 mRNA expressions when SMMC7721 and HepG2 cells were transfected with miR-136-5P mimic or miR-136-5P inhibitor (miR-1365P-in). Transcript levels were normalized to GAPDH expression. ${ }^{* *} \mathrm{P}<0.01$. (B) MiR-136-5P regulated the expression of IRX5 in HCC cells. Relative protein expression levels of IRX5 were detected by Western blot analysis, using GAPDH as an endogenous control. (C) Schematic of wild-type and mutant PGL3-IRX5-3'UTR constructs. (D) Luciferase assays of 293T and SMMC7721 cells transfected with PGL3- IRX5-3'UTR-Wt or PGL3- IRX5-3'UTR-Mut reporter and NC or miR-136-5P mimic. ${ }^{* *} \mathrm{P}<0.01$. (E) qRT-PCR analysis of for CRNDE and miR-136-5P regulated IRX5. Transcript levels were normalized to GAPDH expression. (F) Western blot analysis of CRNDE and miR-136-5P regulated the expression of IRX5, using GAPDH as endogenous control.

Recently, accumulated evidence has shown that a novel regulatory mechanism exists between IncRNAs and miRNAs. LncRNA may function as ceRNA sponges binding to miRNAs and may reciprocally repress the expression and biological functions of miRNAs and their target genes [26-28]. For example, HULC acted as an endogenous sponge to downregulate miR-372 expression in liver cancer 26. TUG1 enhanced tumor-induced angiogenesis and vascular endothelial growth factor expression by directly binding to the miR-299 14 .

To elucidate the molecular mechanism of CRNDE in promoting tumor cell proliferation, migration, and invasion, we performed bioinformatics analysis to explore the potential 


\section{Cellular Physiology Cell Physiol Biochem 2018;50:2229-2248 and BiOChemistry \begin{tabular}{l|l} 
DOl: 10.1159/000495084 & $\begin{array}{l}\text { P } 2018 \text { The Author(s). Published by S. Karger AG, Basel } \\
\text { www.karger.com/cpb }\end{array}$
\end{tabular} \\ Zhu et al.: The Function of CRNDE in Hepatocellular Carcinoma}

targeted gene of CRNDE. First, we found the expression of miR-136-5P decreased in the pcDNA3.1-CRNDE group and miR-136-5P increased in the CRNDE knockdown group (Fig. 4A). The overexpression of miR-136-5P reduced the CRNDE expression, whereas the inhibition of miR-136-5P expression resulted in the significant upregulation of CRNDE (Fig. 4B), suggesting that CRNDE and miR-136-5P could form a reciprocal repression feedback loop. Furthermore, we provided evidence that miR-136-5P targeted CRNDE by directly binding to miRNA-binding sites in CRNDE sequences, and miR-136-5P mimic could abolish the luciferase activity of CRNDE by luciferase assays, suggesting that CRNDE was negatively regulated by miR-136-5P. These data indicated that CRNDE might be a target of miR-136-5P.

MiR-136 decreased in all of the peritoneal metastatic sublines compared with that in the parental line [29]. CRNDE activated the Wnt/ $\beta$-catenin signaling pathway by acting as a molecular sponge of miR-136 in human breast cancer [30]. MiR-136 was downregulated in triple-negative breast cancer and negatively correlated with the World Health Organization grades [31]. Yang et al. determined that miR-136 directly targeted Smad2 and Smad3, leading to reduced migration and invasiveness of lung ADC cell lines, and that miR-136 could play a tumor-suppressive role by repressing epithelial mesenchymal transition and prometastatic traits by targeting Smad2 and Smad3 [32]. In this study, we found that the expression of miR-136-5P increased in HCC tissues and HCC cell lines and that the overexpression of miR136-5P remarkably inhibited proliferation, migration, and invasion in vitro. Conversely, the knockdown of miR-136-5P obtained the opposite results, which was consistent with other studies. Furthermore, our results confirmed that IRX5 was one of the target genes of miR136-5P and that the overexpression of miR-136-5P reduced the expression of IRX5, whereas inhibition of miR-136-5P increased the expression of IRX5. Luciferase assays confirmed that IRX5 was a direct target of miR-136-5P.

Our previous studies found that IncRNAs could modulate the biological behaviors of HCC cells through their neighboring mRNAs $[15,19]$. The neighboring mRNA of CRNDE was IRX5 (Fig. 8A). We also found that the overexpression of CRNDE activated IRX5 expression, and knockdown of CRNDE downregulated IRX5 expression in vitro. The immunohistochemistry assay showed strong expressions of IRX5 in the metastasizing liver and lung of mice in the CRNDE knockdown group in vivo (Fig. 8D). The mechanism of how CRNDE regulates IRX5 in HCC, however, has not been thoroughly elucidated.

Evidence that homeobox genes were involved in the regulation of proliferation through their interaction with several cell cycle regulators, including p21, p27, and cyclin D1, is accumulating [33]. Homeobox genes also appear to play a role in regulating apoptosis, angiogenesis, and metastasis [34]. IRX5 expressions were identified in postnatal bipolar interneurons during retinal development and could play a role in the differentiation of these cells [35]. The upregulated expression of CRNDE indicated a poor prognosis in colorectal cancer, and it was positively correlated with IRX5 mRNA expression [36]. IRX5 was regulated by 1,25-dihydroxyvitamin D3 in human prostate cancer, and it regulated apoptosis and the cell cycle in LNCaP prostate cancer cells [37]. The present study indicated that IRX5 was upregulated in HCC tissues and HCC cell lines and that IRX5 promoted HCC cell proliferation, migration, and invasion in vitro.

In this study, we investigated whether miR-136-5P mediated the suppressive effects of CRNDE in oncogenesis. The results indicated that although the overexpression of CRNDE promoted tumor cell proliferation, migration, and invasion, upregulated miR136-5P could largely reverse these effects (Fig. 11). Furthermore, we found that CRNDE knockdown combined with miR-136-5P overexpression most significantly and reversed IRX5 expression. These data indicated that CRNDE upregulated the expression of IRX5 to promote tumor development by binding miR-136-5P. Therefore, the effect of CRNDE on HCC cells proliferation, migration, and invasion could be explained, although in part, by its function as a molecular sponge of miR-136-5P to upregulate IRX5, which provides a possible mechanism for CRNDE functioning as a tumor oncogene. 


\section{Cellular Physiology Cell Physiol Biochem 2018;50:2229-2248 \begin{tabular}{l|l|l} 
and Biochemistry Published online: 13 November 2018 & $\begin{array}{l}\text { (c) } 2018 \text { The Author(s). Published by S. Karger AG, Basel } \\
\text { www.karger.com/cpb }\end{array}$ \\
\hline
\end{tabular}

\section{Conclusion}

In summary, our data demonstrated that CRNDE acted as a tumor oncogene by exhibiting oncogenic properties of human HCC and revealed a novel CRNDE-miR-136-5PIRX5 regulatory network in HCC. CRNDE may be considered as a potential target for the HCC therapies based on its ability to upregulate IRX5, and it deserves further investigation in the future.

\section{Acknowledgements}

This work was supported by the Major National S\&T Program (2013ZX10002002, ALH), the major project of Chongqing Science \& Technology Commission (cstc2013jcyjC10002, ALH), the Natural Science Foundation Project of CQ CSTC (2010BB5359), the Science and Technology Foundation of Guizhou province (2016 1123), and the Science and Technology Cooperation Projects of Guizhou province (2014 7005). We would like to thank LetPub (www. letpub.com) for providing linguistic assistance during the preparation of this manuscript.

\section{Disclosure Statement}

We declare that all of the authors have not received any financial assistance and have not formed personal relationships with other people or organizations that could inappropriately influence (bias) their work. Examples of potential conflicts of interest include employment, consultancies, stock ownership, honoraria, paid expert testimony, patent applications or registrations, and grants or other funding.

\section{References}

1 Villanueva A, Hernandez-Gea V, Llovet JM: Medical therapies for hepatocellular carcinoma: a critical view of the evidence. Nat Rev Gastroenterol Hepatol 2013;10:34-42.

2 Cech TR, Steitz JA: The noncoding RNA revolution-trashing old rules to forge new ones. Cell. 2014;157:7794.

- 3 Han B, Bu P, Meng X, Hou X: Microarray profiling of long non-coding RNAs associated with idiopathic pulmonary arterial hypertension. Exp Ther Med 2017;13:2657-2666.

4 Lammens T, Durinck K, Wallaert A, Speleman F, Van Vlierberghe P: Long non-coding RNAs in leukemia: biology and clinical impact. Curr Opin Hematol 2017;24:353-358.

5 Salehi S, Taheri MN, Azarpira N, Zare A, Behzad-Behbahani A: State of the art technologies to explore long non-coding RNAs in cancer. J Cell Mol Med 2017;21:3120-3140.

-6 Graham LD, Pedersen SK, Brown GS, Ho T, Kassir Z, Moynihan AT, Vizgoft EK, Dunne R, Pimlott L, Young GP, Lapointe LC, Molloy PL: Colorectal Neoplasia Differentially Expressed (CRNDE), a Novel Gene with Elevated Expression in Colorectal Adenomas and Adenocarcinomas. Genes Cancer 2011;2:829-840.

7 Zhang J, Yin M, Peng G, Zhao Y: CRNDE: An important oncogenic long non-coding RNA in human cancers. Cell Prolif 2018;51:e12440.

8 Hu CE, Du PZ, Zhang HD, Huang GJ: Noncoding RNA CRNDE Promotes Proliferation of Gastric Cancer Cells by Targeting miR-145. Cell Physiol Biochem 2017;42:13-21.

-9 Meng YB, He X, Huang YF, Wu QN, Zhou YC, Hao DJ: Long Non-coding RNA CRNDE Promotes Multiple Myeloma Cell Growth By Suppressing MiR-451. Oncol Res 2017;25:1207-1214.

10 Chen Z, Yu C, Zhan L, Pan Y, Chen L, Sun C: LncRNA CRNDE promotes hepatic carcinoma cell proliferation, migration and invasion by suppressing miR-384. Am J Cancer Res 2016;6:2299-2309.

11 Dong R, Liu XQ Zhang BB, Liu BH, Zheng S, Dong KR: Long non-coding RNA-CRNDE: a novel regulator of tumor growth and angiogenesis in hepatoblastoma. Oncotarget 2017;8:42087-42097. 


\section{Cellular Physiology Cell Physiol Biochem 2018;50:2229-2248

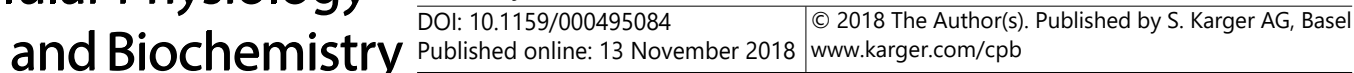

12 Derrien T, Johnson R, Bussotti G, Tanzer A, Djebali S, Tilgner H, Guernec G, Martin D, Merkel A, Knowles DG, Lagarde J, Veeravalli L, Ruan X, Ruan Y, Lassmann T, Carninci P, Brown JB, Lipovich L, Gonzalez JM, Thomas M et al.: The GENCODE v7 catalog of human long noncoding RNAs: analysis of their gene structure, evolution, and expression. Genome Res 2012;22:1775-1789.

13 Salmena L, Poliseno L, Tay Y, Kats L, Pandolfi PP: A ceRNA hypothesis: the Rosetta Stone of a hidden RNA language? Cell 2011;146:353-358.

14 Cai H, Liu X, Zheng J, Xue Y, Ma J, Li Z, Xi Z, Li Z, Bao M, Liu Y: Long non-coding RNA taurine upregulated 1 enhances tumor-induced angiogenesis through inhibiting microRNA-299 in human glioblastoma. Oncogene 2017;36:318-331.

15 Xiong DM, Sheng YR, Ding SJ, Chen J, Tan XX, Zeng T, Qin DD, Zhu LY, Huang AL, Tang H: LINC00052 regulates the expression of NTRK3 by miR-128 and miR-485-3p to strengthen HCC cells invasion and migration. Oncotarget 2016; 7:47593-47608.

16 Zheng J, Liu X, Wang P, Xue Y, Ma J, Qu C, Liu Y: CRNDE Promotes Malignant Progression of Glioma by Attenuating miR-384/PIWIL4/STAT3 Axis. Mol Ther 2016;24:1199-1215.

17 Izaurralde E: Elucidating the temporal order of silencing. EMBO Rep 2012; 13:662-663.

18 Filipowicz W, Bhattacharyya SN, Sonenberg N: Mechanisms of post-transcriptional regulation by microRNAs: are the answers in sight? Nat Rev Genet 2008;9:102-114.

19 Zeng T, Wang D, Chen J, Tian YY, Cai XF, Peng H, Zhu LY, Huang AL, Tang H: LncRNA-AF113014 promotes the expression of Egr2 by interaction with miR-20a to inhibit proliferation of hepatocellular carcinoma cells. PLoS One 2017;12:e177843.

20 He JH, Han ZP, Liu JM, Zhou JB, Zou MX, Lu YB, Li YG, Cao MR: Overexpression of Long Non-coding RNA MEG3 Inhibits Proliferation of Hepatocellular Carcinoma Huh7 Cells via Negative Modulation of miRNA-664. J Cell Biochem 2017;118:3713-3721.

-21 Chen L, Yao H, Wang K, Liu X: Long Non Coding RNA MALAT1 Regulates ZEB1 Expression by Sponging miR14-3p and Promotes Hepatocellular Carcinoma Progression. J Cell Biochem 2017;118:4836-4843.

-22 Xiao JN, Yan TH, Yu RM, Gao Y, Zeng WL, Lu SW, Que HX, Liu ZP, Jiang JH: Long non-coding RNA UCA1 regulates the expression of Snail2 by miR-203 to promote hepatocellular carcinoma progression. J Cancer Res Clin Oncol 2017;143:981-990.

23 Zheng J, Li XD, Wang P, Liu XB, Xue YX, Hu Y, Li Z, Li ZQ, Wang ZH, Liu YH: CRNDE affects the malignant biological characteristics of human glioma stem cells by negatively regulating miR-186. Oncotarget 2015;6:25339-25355.

-24 Shen S, Liu H, Wang Y, Wang J, Ni X, Ai Z, Pan H, Liu H, Shao Y: Long non-coding RNA CRNDE promotes gallbladder carcinoma carcinogenesis and as a scaffold of DMBT1 and C-IAP1 complexes to activating PI3KAKT pathway. Oncotarget 2016;7:72833-72844.

-25 Esposti DD, Hernandez-Vargas H, Voegele C, Fernandez-Jimenez N, Forey N, Bancel B, Le Calvez-Kelm F, McKay J, Merle P, Herceg Z: Identification of novel long non-coding RNAs deregulated in hepatocellular carcinoma using RNA-sequencing. Oncotarget 2016;7:31862-31877.

26 Wang J, Liu X, Wu H, Ni P, Gu Z, Qiao Y, Chen N, Sun F, Fan Q: CREB up-regulates long non-coding RNA, HULC expression through interaction with microRNA-372 in liver cancer. Nucleic Acids Res 2010;38:5366-5383.

-27 Wang P, Liu YH, Yao YL, Li Z, Li ZQ, Ma J, Xue YX: Long non-coding RNA CASC2 suppresses malignancy in human gliomas by miR-21. Cell Signal 2015;27:275-282.

28 Li T, Meng XL, Yang WQ: Long Noncoding RNA PVT1 Acts as a "Sponge” to Inhibit microRNA-152 in Gastric Cancer Cells. Dig Dis Sci 2017;62:3021-3028.

29 Zheng J, Ge P, Liu X, Wei J, Wu G, Li X: MiR-136 inhibits gastric cancer-specific peritoneal metastasis by targeting HOXC10. Tumour Biol 2017;39:1010428317706207.

-30 Huan J, Xing L, Lin Q Xui H, Qin X: Long noncoding RNA CRNDE activates Wnt/beta-catenin signaling pathway through acting as a molecular sponge of microRNA-136 in human breast cancer. Am J Transl Res 2017;9:1977-1989.

-31 Yan M, Li X, Tong D, Han C, Zhao R, He Y, Jin X: miR-136 suppresses tumor invasion and metastasis by targeting RASAL2 in triple-negative breast cancer. Oncol Rep 2016;36:65-71.

32 Yang Y, Liu L, Cai J, Wu J, Guan H, Zhu X, Yuan J, Chen S, Li M: Targeting Smad2 and Smad3 by miR-136 suppresses metastasis-associated traits of lung adenocarcinoma cells. Oncol Res 2013;21:345-352.

-33 Del Bene F, Wittbrodt J: Cell cycle control by homeobox genes in development and disease. Semin Cell Dev Biol 2005;16:449-460. 


\section{Cellular Physiology Cell Physiol Biochem 2018;50:2229-2248 \begin{tabular}{l|l|l} 
and Biochemistry & $\begin{array}{l}\text { DOl: 10.1159/000495084 } \\
\text { Published online: } 13 \text { November } 2018\end{array}$ & $\begin{array}{l}\text { O } 2018 \text { The Author(s). Published by S. Karger AG, Basel } \\
\text { www.karger.com/cpb }\end{array}$ \\
\hline
\end{tabular} \\ Zhu et al.: The Function of CRNDE in Hepatocellular Carcinoma}

-34 Lohmann I, McGinnis N, Bodmer M, McGinnis W: The Drosophila Hox gene deformed sculpts head morphology via direct regulation of the apoptosis activator reaper. Cell 2002;110:457-466.

-35 Cheng CW, Chow RL, Lebel M, Sakuma R, Cheung HO, Thanabalasingham V, Zhang X, Bruneau BG, Birch DG, Hui CC, McInnes RR, Cheng SH: The Iroquois homeobox gene, Irx5, is required for retinal cone bipolar cell development. Dev Biol 2005;287:48-60.

-36 Liu T, Zhang X, Yang YM, Du LT, Wang CX: Increased expression of the long noncoding RNA CRNDE-h indicates a poor prognosis in colorectal cancer, and is positively correlated with IRX5 mRNA expression. Onco Targets Ther 2016;9:1437-1448.

-37 Myrthue A, Rademacher BL, Pittsenbarger J, Kutyba-Brooks B, Gantner M, Qian DZ, Beer TM: The iroquois homeobox gene 5 is regulated by 1,25-dihydroxyvitamin D3 in human prostate cancer and regulates apoptosis and the cell cycle in LNCaP prostate cancer cells. Clin Cancer Res 2008;14:3562-3570. 